\title{
Impact of Nicotine Exposure on Hair Cell Toxicity and Embryotoxicity During Zebrafish Development
}

\author{
Myung Hoon Yoo $\cdot$ Yoon Chan Rah² $\cdot$ Saemi Park $\cdot$ Soonil Koun ${ }^{3} \cdot$ Gi Jung $\mathrm{Im}^{2} \cdot$ Sung Won Chae ${ }^{2}$ \\ Hak Hyun Jung ${ }^{2} \cdot$ June Choi $^{2}$ \\ ${ }^{1}$ Department of Otorhinolaryngology-Head and Neck Surgery, Kyungpook National University School of Medicine, Daegu; \\ ${ }^{2}$ Department of Otorhinolaryngology-Head and Neck Surgery, Korea University College of Medicine, Seoul; \\ ${ }^{3}$ Biomedical Research Center, Korea University Ansan Hospital, Ansan, Korea
}

Objectives. Nicotine has various adverse effects including negative impacts associated with maternal exposure. In the current study, we examined nicotine-induced damage of hair cells and embryotoxicity during zebrafish development.

Methods. Zebrafish embryos were exposed to nicotine at several concentrations $(5,10,20$, and $40 \mu \mathrm{M})$ and embryotoxicity were evaluated at 72 hours, including hatching rate, mortality, teratogenicity rate, and heart rate. Hair cells within the supraorbital (SO1 and SO2), otic (O1), and occipital (OC1) neuromasts were identified at 120 hours. Apoptosis and mitochondrial damage of hair cells were analyzed using TUNEL (terminal deoxynucleotidyl transferase-mediated dUTP-biotin nick end labeling) and DASPEI (2-[4-(dimethylamino)styryl]-N-ethylpyridinium iodide) assays, respectively, and changes of ultrastructure were observed by scanning electron microscopy.

Results. The control group without nicotine appeared normal with overall mortality and teratogenicity rate $<5 \%$. The hatching rate and mortality rate was not significantly different according to nicotine concentration ( $\mathrm{n}=400 \mathrm{each}$ ). The abnormal morphology rate $(n=400)$ increased and heart rate $(n=150)$ decreased with increasing nicotine concentration $(P<0.05)$. Nicotine-induced hair cell damage significantly increased as nicotine concentration increased. A significantly greater number of TUNEL-positive cells $(P<0.01)$ and markedly smaller DASPEI area $(P<0.01)$ were shown as nicotine concentration increased.

Conclusion. The current results suggest that nicotine induces dose-dependent hair cell toxicity in embryos by promoting apoptosis and mitochondrial and structural damage.

Keywords. Nicotine; Tobacco; Zebrafish; Hair Cells; Ototoxicity; Embryotoxicity

\section{INTRODUCTION}

Tobacco smoking is an important environmental health problem that is associated with many human diseases [1]. Maternal smoking increases the risk of various complications, ranging from miscarriage to fetal brain development [2]. Among more

\footnotetext{
- Received July 15, 2017

Revised October 19, 2017

Accepted November 10, 2017

- Corresponding author: June Choi

Department of Otorhinolaryngology-Head and Neck Surgery, Korea

University Ansan Hospital, Korea University College of Medicine,

123 Jeokgeum-ro, Danwon-gu, Ansan 15355, Korea

Tel: +82-31-412-5170, Fax: +82-31-412-5174

E-mail: mednlaw@korea.ac.kr
}

than 7,000 chemicals in tobacco, nicotine is undoubted one of the most widely studied agents. Nicotine acts through the stimulation of nicotinic acetylcholine receptors (nAChRs) located on neuronal or non-neuronal tissues [3]. In addition, these receptors are represented during development, and prenatal maternal nicotine exposure results in diverse adverse effects in various animal models [2,3].

Zebrafish (Danio rerio) is known to be an effective model for the research of embryotoxicity because of its ex utero embryo development, transparency of embryos, and short seeding period $[4,5]$. Since nAChRs are confirmed to be represented in zebrafish embryos, and regulate nicotine-induced changes in embryonic morphology, zebrafish are appearing as a useful model for conducting the molecular and biological effects of nicotine

Copyright (C) 2018 by Korean Society of Otorhinolaryngology-Head and Neck Surgery.

This is an open-access article distributed under the terms of the Creative Commons Attribution Non-Commercial License (http://creativecommons.org/licenses/by-nc/4.0)

which permits unrestricted non-commercial use, distribution, and reproduction in any medium, provided the original work is properly cited. 
exposure [5-7]. In zebrafish, embryonic nicotine exposure results in various development problems including growth retardation [8], altered neural development [5], cardiac toxicity [9], muscle development alteration [10], and behavior abnormality [2,5].

Although data on the association of smoking and hearing loss have been in contrast to human adult cohort studies [11,12], smoking was known to deteriorate the auditory thresholds at high frequencies [13] and cause lower response levels in transient evoked otoacoustic emissions [14]. In particular, prenatal or childhood nicotine exposure affects hearing in humans [1417]. In addition, nicotine administration in adult guinea pigs caused damage of outer hair cells, especially at the stereocilia, and expansion of the surrounding supporting cells [18]. However, the hair cell damage by prenatal nicotine exposure has not yet been entirely established until recently. In the current study, we assessed the in vivo hair cell toxicity and embryotoxicity of nicotine during zebrafish development.

\section{MATERIALS AND METHODS}

\section{Chemicals and zebrafish preparation}

The (-)-nicotine (product no. N3876) used in this study was acquired from Sigma-Aldrich (St. Louis, MO, USA). Nicotine solutions were prepared in distilled water and diluted in embryo medium to achieve final concentrations. In addition, this solution was produced daily as needed for all experiments.

Wild-type (AB) and transgenic (Tg; brn3c:EGFP and cmlc2: EGFP) zebrafish were sustained at $28.5^{\circ} \mathrm{C}$ under a 14 hours light and 10 hours dark cycle in a zebrafish facility at Korea University Ansan Hospital as previously described $[7,19]$. Newly hatched brine shrimp (Artemia; San Francisco Bay Brand Inc., Newark, CA, USA) were supplied two times per day for the adult fish. Tg (brn3c:EGFP) and Tg (cmlc2:EGFP) express green fluorescence characteristically in hair cells and myocardial cells, allowing evaluation of heart size, respectively. The wild type and $\mathrm{Tg}$ zebrafish larvae were produced by paired matings of agematched adult fish, and larvae were sustained at a density of approximately 50 embryos per 100 -mm petri dish in embryo media as previously described [20].

Our study was permitted by Institutional Animal Care and Use Committee of Korea University Ansan Hospital (approval No. KUIACUC-2015-173). All tests were performed within the guidelines of the Animal Care Ethics Committee of Korea Uni-

\section{H I G H L I G H T S}

- Zebrafish is an effective model for the study of embryotoxicity.

- Nicotine can affect embryogenesis during zebrafish development.

- Nicotine induce dose-dependent hair cell toxicity in embryos. versityand the National Institutes of Health guidelines.

\section{Assessment of embryotoxicity in zebrafish}

Larvae (wild-type AB strain) were seeded in a 6-well plate (5 $\mathrm{mL}$ volume for each well) with different concentrations of nicotine $(0,5,10,20$, and $40 \mu \mathrm{M})$ and then incubated at $28.5^{\circ} \mathrm{C} \pm$ $1^{\circ} \mathrm{C}$ for 72 hours (10 larvae per well; 80 larvae per treatment concentration; total $n=400$ ). Because the stability of nicotine in embryo media is not determined, the nicotine-containing embryo media was replaced daily. All assessments of embryotoxicity were performed at 72 hours post-fertilization (hpf) using microscope (LUMAR V12, Carl Zeiss, Jena, Germany; 6.4-80× magnification, AxioVision 4.8.2, Carl Zeiss) as previously described [4].The hatching rate and the mortality rate were calculated from the number of hatched larvae and the number of dead larvae divided by the number of total larvae, respectively. Teratogenicity rate was counted from number of fish showing systemic teratogenicities including bending of the spine and tail, stunted growth, malformed yolk sacs, and edema in the body cavity. In addition, the rate of total abnormal findings (mortality rate+teratogenicity rate) was also demonstrated. For evaluation of heart malformation, Tg (cmlc2:EGFP) zebrafish morphology was checked at $72 \mathrm{hpf}$. Also, heart rate at $72 \mathrm{hpf}$ was scored real-time by direct microscopic inspection for 60 seconds at each concentration of nicotine ( $\mathrm{n}=30$ for each group), while larvae were anesthetized with tricaine $(200 \mu \mathrm{L})$.

\section{Assessment of hair cell toxicity in zebrafish}

The wild-type and Tg (brn3c:EGFP) larvae were revealed to nicotine at 5, 10, 20, and $40 \mu \mathrm{M}$ for 120 hours. At $120 \mathrm{hpf}$, the larvae were rinsed with embryo medium three times and anesthetized with a tricaine (3-aminobenzoic acid 0.4 g/ethyl ester; 100 $\mathrm{mL} ; \mathrm{pH} 7$, adjusted using Tris buffer) for 5 minutes as previously described [19].

In the Tg (brn3c:EGFP) larvae, hair cells in four neuromasts (supraorbital [SO1 and SO2], otic [O1], and occipital [OC1]) were analyzed on one side of each fish under a fluorescence microscope (AxioCam MRc5, Carl Zeiss; 5, 10, 20, 40× magnification, AxioVision 4.8.2). The total number of hair cells within four neuromasts (three times replicated with $n=30$ for each group) was analyzed. Furthermore, apoptosis of hair cell was identified at $120 \mathrm{hpf}$ in Tg (brn3c:EGFP) zebrafish by terminal deoxynucleotidyl transferase-mediated dUTP-biotin nick end labeling (TUNEL) staining with an in situ cell detection kit (Roche Molecular Biochemicals, Mannheim, Germany) in accordance with the manufacturer's protocol. TUNEL-positive cells were analyzed in four neuromasts in 10 fish from each group (total $n=50$ ), as previously described [19].

To evaluate mitochondrial damage within the hair cells, the fluorescent dye, 2-(4-[dimethylamino]styryl)-N-ethylpyridinium iodide (DASPEI; Invitrogen, Carlsbad, CA, USA) was applied to wild-type larvae. After anesthetization, larvae were incubated in 
embryo medium containing $0.005 \%$ DASPEI for 15 minutes. The DASPEI-stained area within SO1, SO2, O1, and OC1 was examined under a fluorescence microscope and measured using Image J (ver. 1.48; National Institutes of Health, Bethesda, MA, USA) as we previously described [20]. The average area of DASPEI staining in neuromasts was compared among all experimental groups and the control group ( $\mathrm{n}=6$ fish for each group).

\section{Measurement of intracellular reactive oxygen species generation}

To examine whether the reactive oxygen species (ROS) production is influenced by nicotine exposure, we evaluated intracellular ROS generation using an oxidation-sensitive fluorescent probe dye; a 2,7-dichlorodihydrofluorescein diacetate (DCHFDA). Wild-type zebrafish were washed three times with embryo media, and seeded into 24-well plates. And then, DCHF-DA was treated with $10 \mu \mathrm{M}$ for 5 minutes, and washed three times in embryo media. For DNA staining, 4',6-diamidino-2-phenylindole (DAPI) was applied for 3 minutes in $0.1 \mathrm{mg} / \mathrm{mL}$. After washing again, groups were subdivided for normal control, positive control (125 $\mu \mathrm{M}$ neomycin for 20 minutes), and two experimental groups treated with $5 \mu \mathrm{M}$ and $40 \mu \mathrm{M}$ nicotine (for 20 minutes). After the treatments, larvae were anesthetized and mounted in $2 \%$ methyl cellulose for observation under a fluorescence microscope.

\section{Scanning electron microscopy}

The 5-dpf wild-type larvae exposed to $5 \mu \mathrm{M}$ and $40 \mu \mathrm{M}$ nicotine and normal controls were prefixed by immersion in $2 \%$ glutaraldehyde in $0.1 \mathrm{M}$ phosphate buffer and postfixed for 2 hours in $1 \%$ osmic acid dissolved in phosphate-buffered saline. Larvae were managed in a graded series of ethanol and t-butyl alcohol, dehydrated in a freeze dryer (ES-2030; Hitachi, Tokyo, Japan), platinum coated using an ion coater (IB-5; Eiko, Tokyo, Japan) and investigated using scanning electron microscopy (SEM; S-4700; 2,500, 3,000, 5,000 $\times$ magnification, Hitachi) as previously described [20].

\section{Statistical analysis}

Statistical comparisons were performed using likelihood ratio test for trend for categorical data including hatching rate, mortality rate, teratogenicity rate, and total abnormal findings. The one-way analysis of variance (ANOVA) was used for multiple comparisons of numerical data and a post-hoc analysis was per-
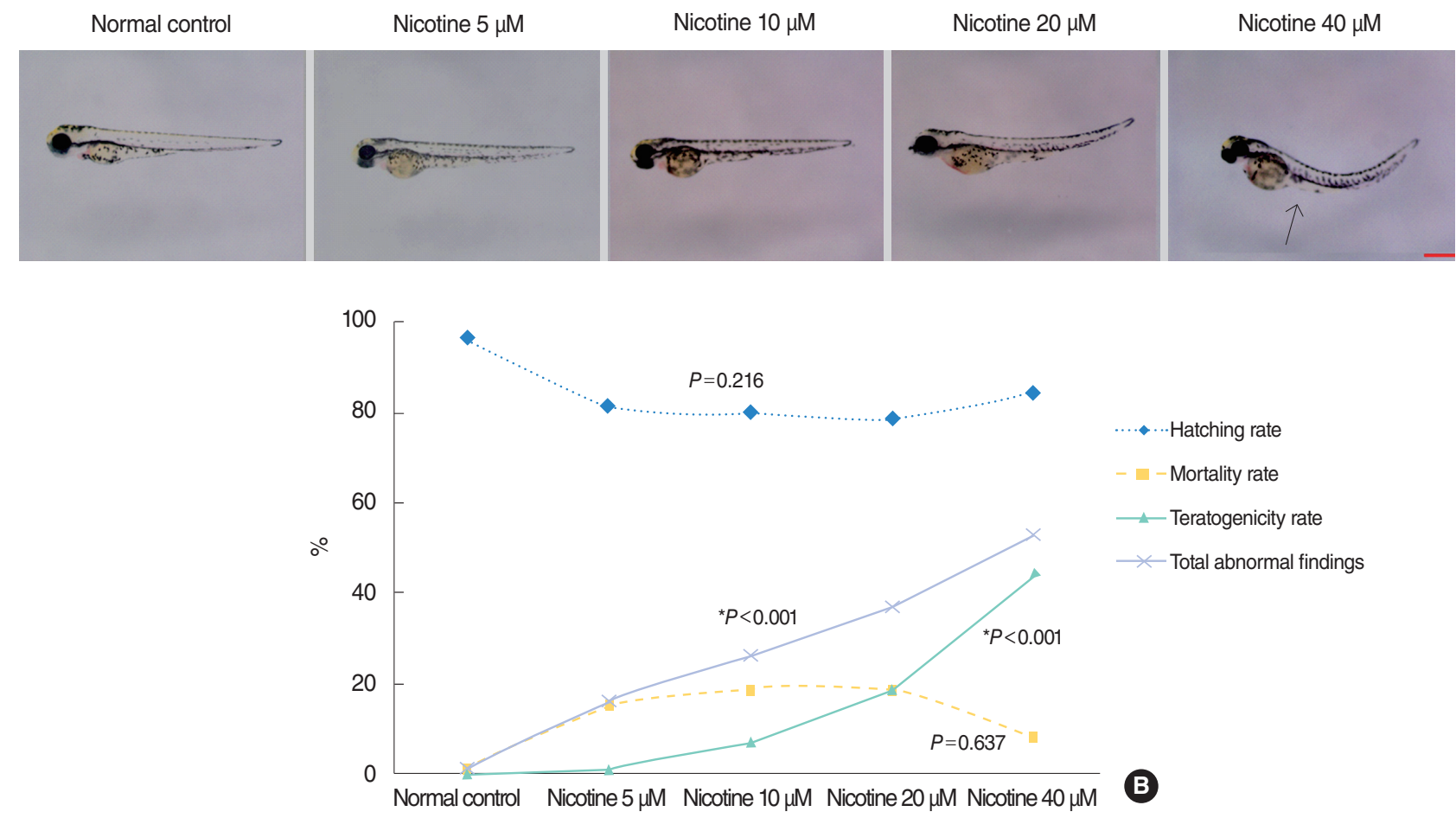

Fig. 1. Nicotine-induced embryotoxicity at 72 hours post-fertilization (hpf). (A) Hatching/non-hatching embryo findings. The number of embryos showing abnormal morphology tended to be increased in the nicotine-exposed groups (arrow: bending of the trunk). All images were captured at $72 \mathrm{hpf}(\times 32)$. Scale bar $=1,000 \mu \mathrm{m}$. (B) Hatching rate, mortality rate, teratogenicity rate, and total abnormal findings (mortality rate+teratogenicity rate) showed statistically significant differences among nicotine concentrations ( $n=100$ per concentration, linear by linear association). Nicotine-exposed groups showed a lower hatching rate and higher mortality and teratogenicity than the control group. The rate of teratogenicity and total abnormal findings increased in a dose-dependent manner (1\% in the normal control group, $16 \%$ for $5 \mu \mathrm{M}$ nicotine, $26 \%$ for $10 \mu \mathrm{M}$ nicotine, $37 \%$ for $20 \mu \mathrm{M}$ nicotine, and $53 \%$ for $40 \mu \mathrm{M}$ nicotine; (total $\mathrm{n}=400$ ). *Statistically significant. All data were evaluated at $72 \mathrm{hpf}$. 
formed using Tukey's honestly significant difference test. In the current study, statistical analysis was conducted with IBM SPSS ver. 20.0 (IBM Corp., Armonk, NY, USA). A $P<0.05$ were considered statistically significant.

\section{RESULTS}

Embryotoxicity of nicotine in zebrafish (brn3c and cmcl2)

The control group without nicotine exposure appeared normal with an overall mortality and teratogenicity rate $<5 \%$, and
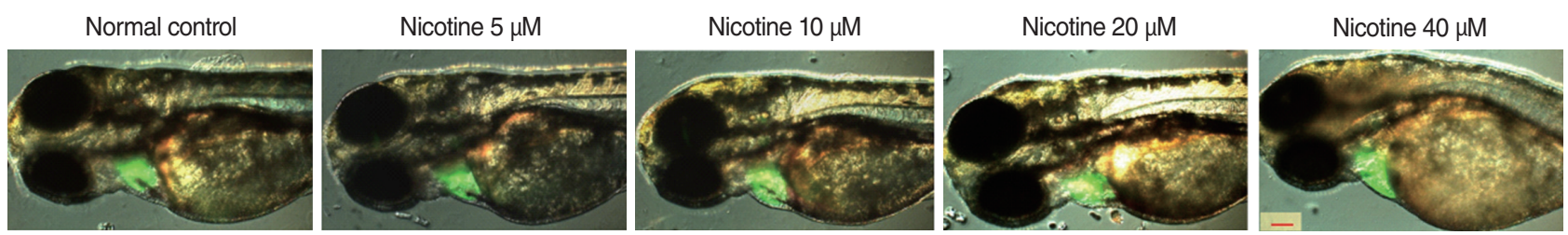

A

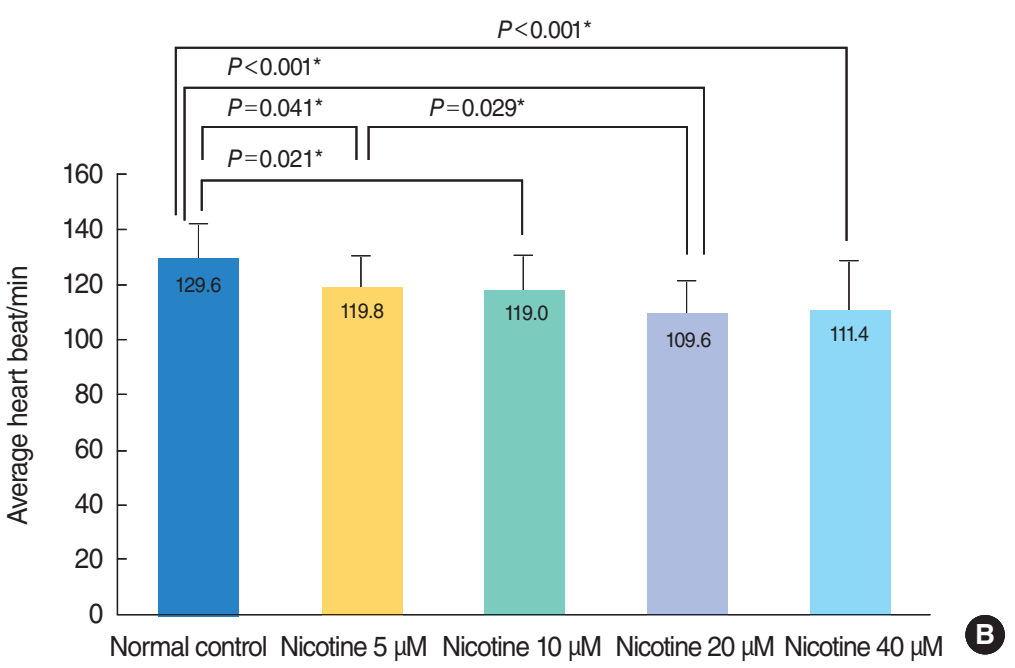

Fig. 2. Heart morphology evaluation and changes in heart rate induced by nicotine treatment. (A) Merged images from the fluorescent image and optical image are shown. There was no difference in heart size between groups (green area). All images were captured using transgenic (cmlc2:EGFP) zebrafish at 72 hours post-fertilization (hpf, $\times 16)$. Scale bar $=200 \mu \mathrm{m}$. (B) The heart rate of zebrafish embryos was significantly decreased in nicotine-exposed groups $(P<0.001$, one-way analysis of variance). All data were evaluated at 72 hpf. Only statistically significant pair-wise comparisons in post-hoc analysis are shown. *Statistically significant $(P<0.05$, total $n=150)$.
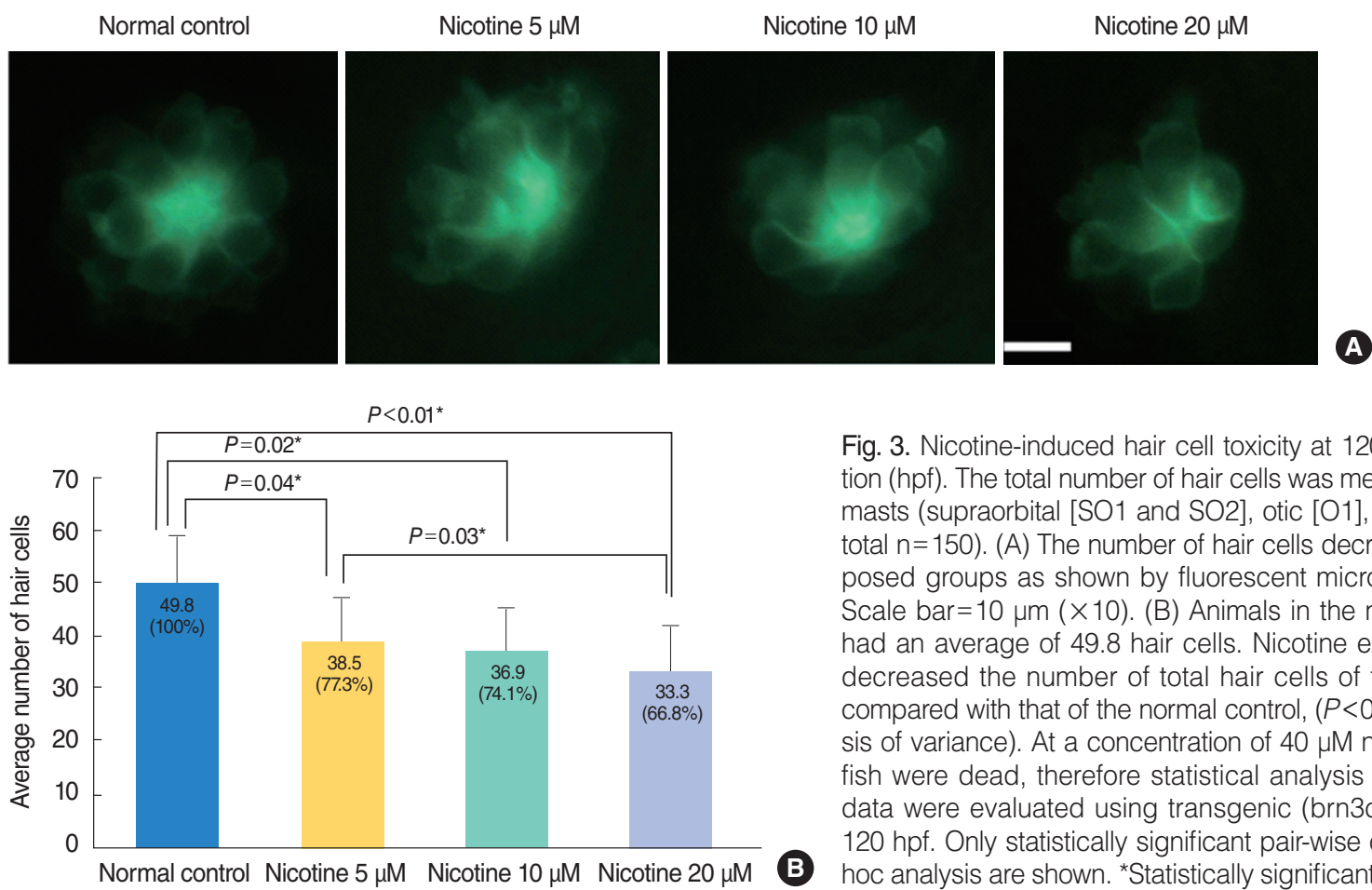

Fig. 3. Nicotine-induced hair cell toxicity at 120 hours post-fertilization (hpf). The total number of hair cells was measured in four neuromasts (supraorbital [SO1 and SO2], otic [O1], and occipital [OC1]; total $n=150$ ). (A) The number of hair cells decreased in nicotine-exposed groups as shown by fluorescent microscopy (OC1, $\times 40$ ). Scale bar $=10 \mu \mathrm{m}(\times 10)$. (B) Animals in the normal control group had an average of 49.8 hair cells. Nicotine exposure significantly decreased the number of total hair cells of the four neuromasts compared with that of the normal control, $(P<0.001$, one-way analysis of variance). At a concentration of $40 \mu \mathrm{M}$ nicotine almost all the fish were dead, therefore statistical analysis was unavailable. All data were evaluated using transgenic (brn3c:EGFP) zebrafish at $120 \mathrm{hpf}$. Only statistically significant pair-wise comparisons in posthoc analysis are shown. * Statistically significant $(P<0.05)$. 
hatching rate of $96 \%$ (Fig. 1). The hatching rate was not significantly different according to nicotine concentration $(P=0.216$, linear by linear association). At $72 \mathrm{hpf}, 81 \%$ of embryos hatched in the $5 \mu \mathrm{M}$ nicotine group compared with $84 \%$ in the group treated with $40 \mu \mathrm{M}$ (Fig. 1). The mortality rate was also not significantly different between the normal control and nicotinetreated groups ( $P=0.637$, linear by linear association) (Fig. 1). The mortality rate was $1 \%, 15 \%, 19 \%, 19 \%$, and $9 \%$ in control, nicotine 5, 10, 20, and $40 \mu \mathrm{M}$ groups, respectively. With increasing nicotine concentration, the teratogenicity rate markedly increased $(P<0.001$, linear by linear association) (Fig. 1). The teratogenicity rate was $1 \%$ in the $5 \mu \mathrm{M}$ nicotine group and $44 \%$ in the $40 \mu \mathrm{M}$ nicotine group. Furthermore, the rate of total abnormal findings notably increased in a dose-dependent manner $(P<0.001$, linear by linear association) (Fig. 1). The results of embryotoxicity for different treatment concentrations of nicotine are presented in Fig. 1.

No significant heart malformation was observed in nicotineexposed embryos of Tg ( $\mathrm{cmcl} 2$ ) (Fig. 2A). The heart rate significantly decreased as nicotine concentration increased $(P<0.001$, n=30 for each concentration, ANOVA) (Fig. 2B).

\section{Nicotine-induced hair cell toxicity in neuromasts of zebrafish embryos (brn3c)}

Nicotine-induced hair cell damage in four neuromasts of $\mathrm{Tg}$ (brn3c:EGFP) embryos was evaluated (Fig. 3). The average number of hair cells in four neuromasts significantly decreased as nicotine concentration increased (control, $100 \%$; nicotine $5 \mu \mathrm{M}$, $77.3 \%$; nicotine $10 \mu \mathrm{M}, 74.1 \%$; nicotine $20 \mu \mathrm{M}, 66.8 \%$; $P<0.05$, ANOVA). Fish exposed to $40 \mu \mathrm{M}$ nicotine were almost all dead at $120 \mathrm{hpf}$, therefore comparison with other groups was not possible. As shown in Fig. 4, significantly more TUNEL-positive cells $(P<0.001$, ANOVA) were observed in Tg (brn3c:EGFP) embryos as nicotine concentration increased.

\section{DASPEI staining in wild-type embryos}

The average DASPEI-stained area in four neuromasts was analyzed among all experimental groups and the control group ( $n=6$ for each concentration). Treatment of wild-type zebrafish with nicotine significantly increased the amount of cellular dam-
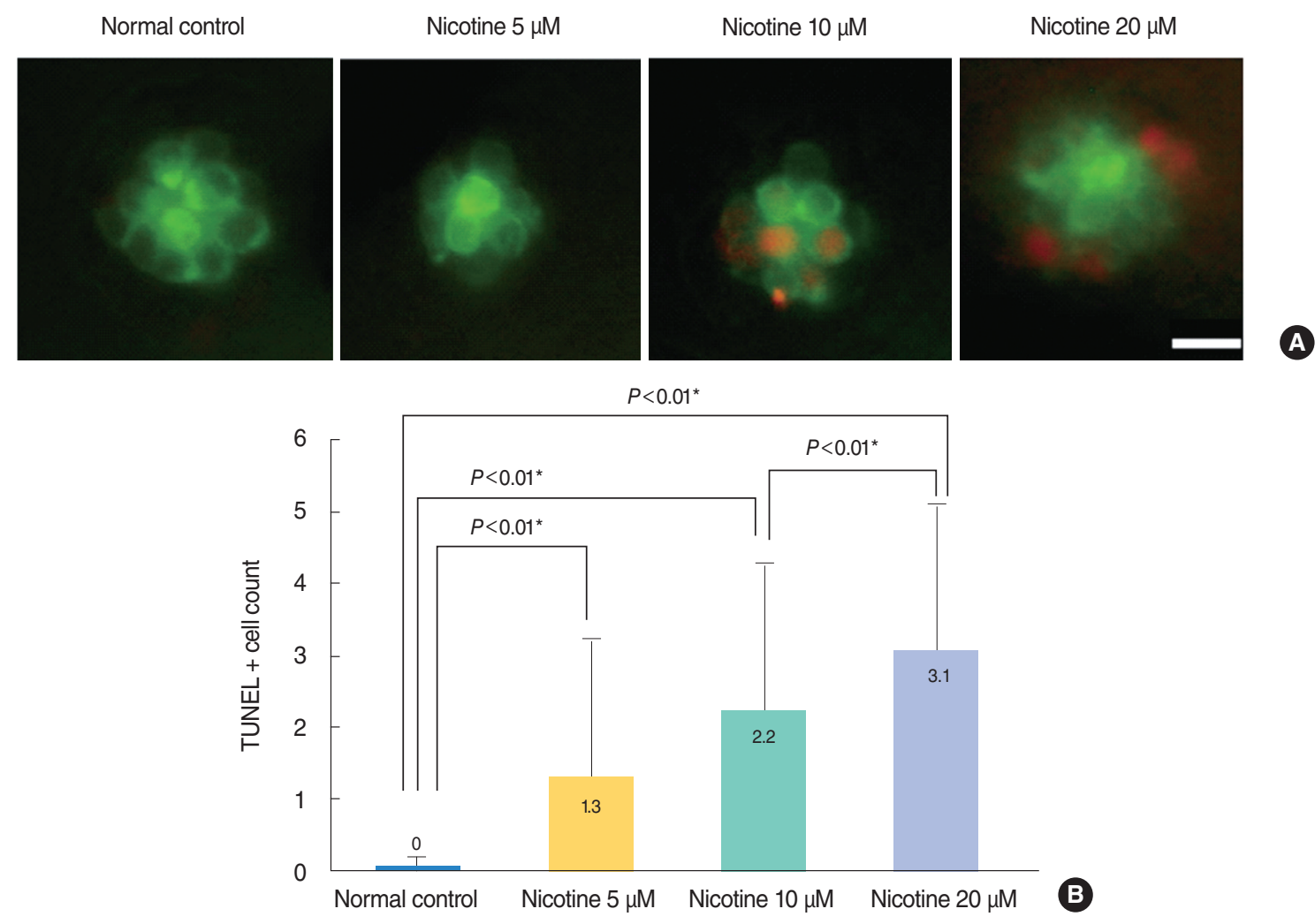

Fig. 4. Nicotine-induced apoptosis in hair cells within neuromasts. Nicotine-induced apoptosis was confirmed by terminal deoxynucleotidyl transferase-mediated dUTP-biotin nick end labeling (TUNEL) assay. (A) Green dots represent hair cells. Apoptotic cells appear as light red dots in the merged image obtained by fluorescent microscopy after the TUNEL reaction. All images were obtained using transgenic (brn3c:EGFP) zebrafish at 120 hours post-fertilization. Scale bar=10 $\mu \mathrm{m}(\times 40)$. (B) The average number of TUNEL-positive cells was measured in each neuromast (supraorbital [SO1, SO2], otic [01], and occipital [OC1]; total n=50). At a concentration of $40 \mu \mathrm{M}$, the fish were almost all dead and statistical analysis was unavailable. The number of TUNEL-positive cells was significantly increased in nicotine-exposed groups $\left(P<0.001\right.$, one-way analysis of variance). Only statistically significant pair-wise comparisons in post-hoc analysis are shown. ${ }^{\star}$ Statistically significant $(P<0.05)$. 

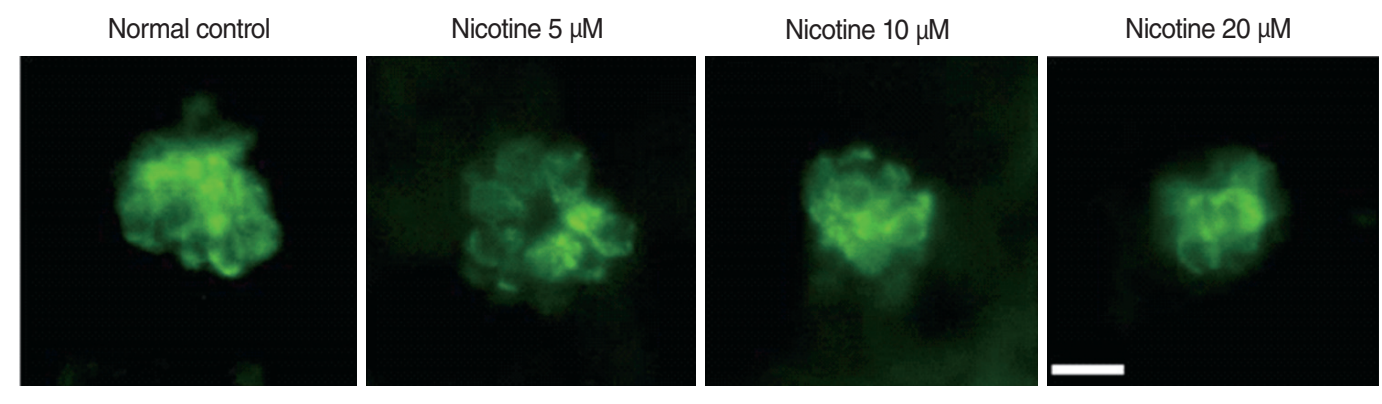

A

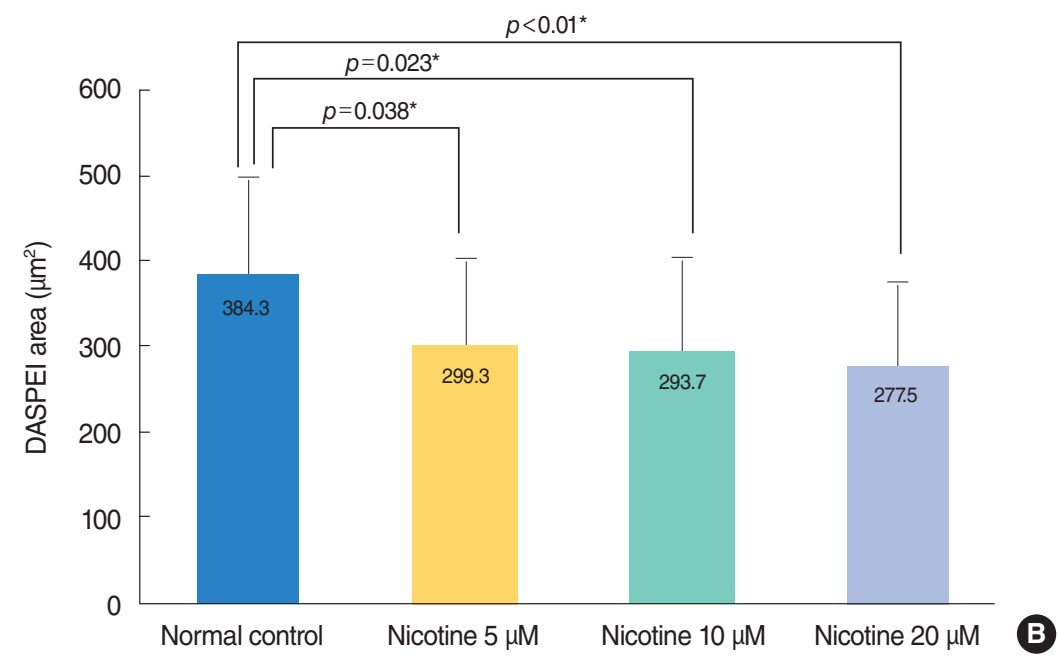

Fig. 5. Analysis of hair cell damage by 2-(4-[dimethylamino]styryl)-N-ethylpyridinium iodide (DASPEI) assay. (A) Staining of hair cells with DASPEl showed that treatment with nicotine decreased the number of hair cells in neuromasts. Scale bar $=10 \mu \mathrm{m}(\times 40)$. $(B)$ Average DASPEIstained area in four neuromasts (supraorbital [SO1, SO2], otic [01], and occipital [OC1]; total $n=30$ ) significantly decreased as nicotine concentration increased $(P<0.001$, one-way analysis of variance). Fish exposed to $40 \mu \mathrm{M}$ nicotine were almost all dead after 120 hours post-fertilization. Only statistically significant pair-wise comparisons in post-hoc analysis are shown. *Statistically significant $(P<0.05)$.

age, as shown by a decrease in the average DASPEI-stained area expressed in hair cells $(\mathrm{n}=30, P<0.001$, ANOVA) (Fig. 5). The average DASPEI area in four neuromasts was $384.3 \pm 113.9 \mu \mathrm{m}^{2}$ in the control group, compared with $277.5 \pm 101.1 \mu \mathrm{m}^{2}$ for fish treated with $20 \mu \mathrm{M}$ nicotine. As in the TUNEL assay, fish treated with $40 \mu \mathrm{M}$ nicotine were almost all dead after $120 \mathrm{hpf}$.

\section{Measurement of intracellular ROS generation in wild-type larvae}

Representative result of ROS experiment is shown in Fig. 6. Positive control (125 $\mu \mathrm{M}$ neomycin) showed increased ROS in hair cells using DCHF-DA staining. In $5 \mu \mathrm{M}$ nicotine, ROS generation was not different when compared with normal control, but was definitely more expressed in $40 \mu \mathrm{M}$ nicotine.

\section{Nicotine-induced ultrastructural changes by SEM}

When observed with SEM, the kinocilia and the stereocilia bundles of hair cells were intact in the control group; however, the kinocilia of hair cells were destroyed in the $40 \mu \mathrm{M}$ nicotine group (Fig. 7) and the $5 \mu \mathrm{M}$ nicotine group showed fewer stereocilia bundles than the control group.

\section{DISCUSSION}

Nicotine is known to act through the activation of nAChRs, which can induce cellular apoptosis [21]. Previous studies reported that hair cells have nAChRs that are activated by the neurotransmitter acetylcholine, and that these nAChRs are crucial for establishment of innervation to the hair cells prior to hearing [18].

nAChRs are reported to be expressed in zebrafish embryos, and acetylcholinesterase-positive cells have been known to be essential for normal neuromuscular and neuronal development $[5,22]$. Because nicotine induces alterations in embryonic morphology, the zebrafish model has been used in various studies investigating the molecular and biological effects of nicotine exposure [5-7]. Several zebrafish nACHRs subunits are characterized in early development, and they have a high degree of sequence identity to nACHRs expressed in mammalian species [23]. The effects of prenatal nicotine exposure have been widely studied using zebrafish, and the zebrafish model is also used in various research studies investigating nicotine addiction, cognitive function, anxiolytic effects, and nAChR characterization 


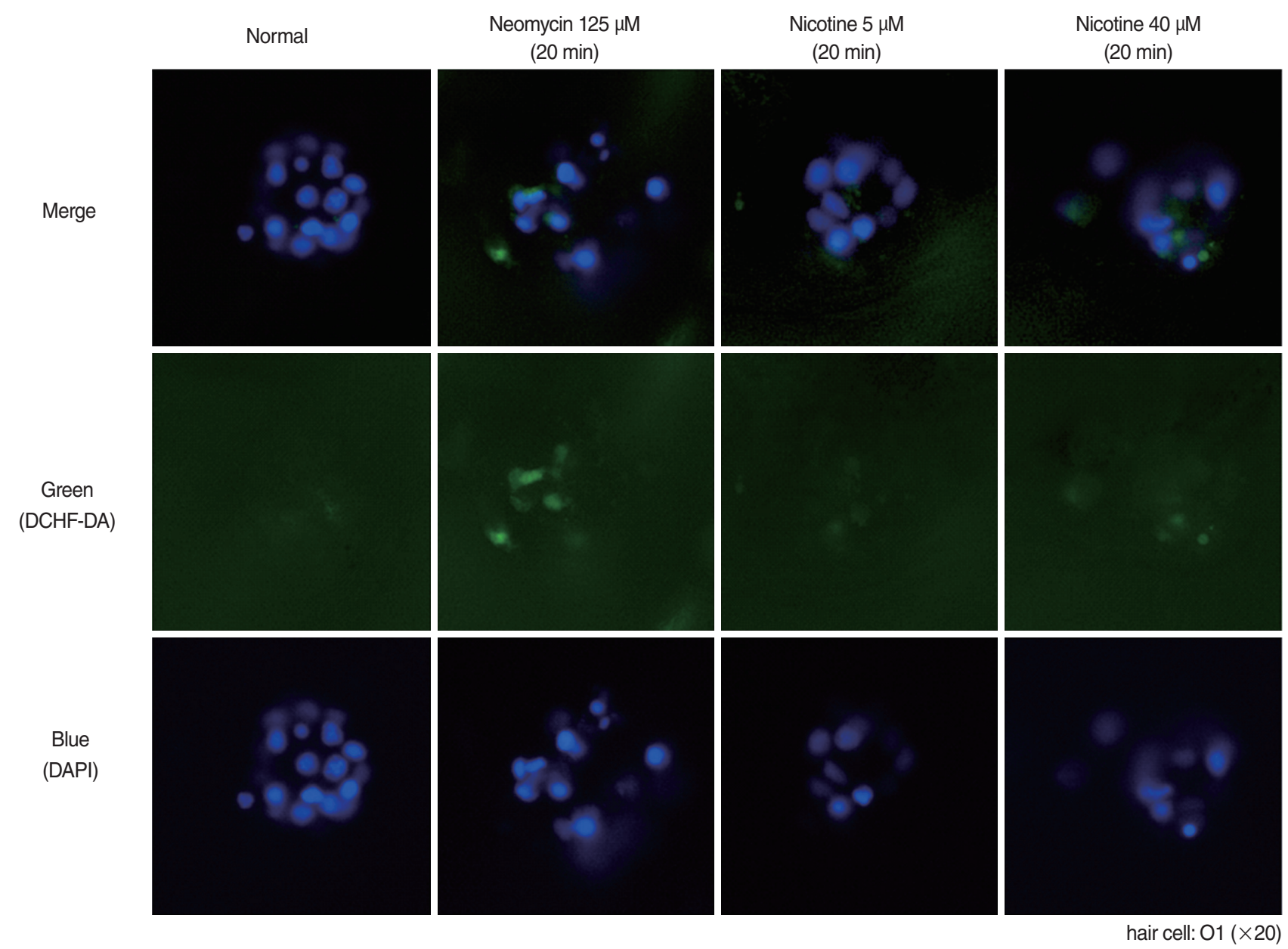

Fig. 6. Reactive oxygen species (ROS) production by 2,7-dichlorodihydrofluorescein diacetate (DCHF-DA) assay. For evaluation of ROS generation, wild type zebrafish was used with DCHF-DA assay on hair cells as in green dots in the middle row. A 4',6-diamidino-2-phenylindole (DAPI)-positive cells were observed as blue fluorescent dots as in the lower row. By merging those figures, the ROS production cells could be clearly identified as in the upper row. Neomycin as positive control showed strongest ROS generation. Despite not strong as in neomycin, there was increased ROS generation in hair cells in nicotine $40 \mu \mathrm{M}$. 01, otic.
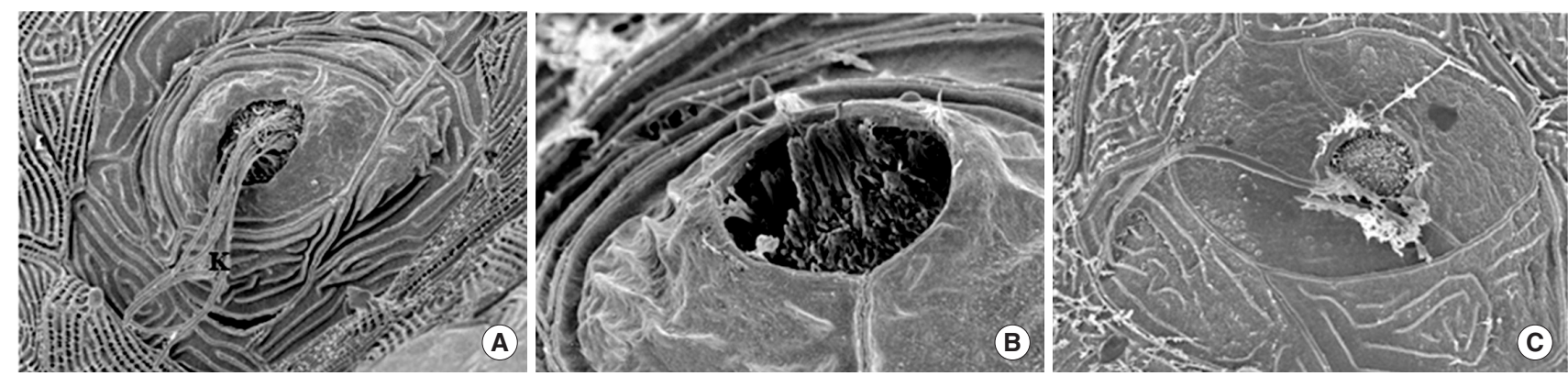

Fig. 7. Scanning electron microscopy. When transgenic (brn3c:EGFP) zebrafish were exposed to nicotine for 120 hours, the kinocilia of hair cells in neuromasts were clearly observed in the normal control (A) and $5 \mu \mathrm{M}$ nicotine group (B), but not in the $20 \mu \mathrm{M}$ nicotine group (C). All images were obtained in three zebrafish at 120 hours post-fertilization for each group. Scale bar (at the bottom of each figure, one space) $=5$ or $10 \mu \mathrm{m}$.

\section{$[5,24]$.}

In the present work, nicotine administration induced hair cell toxicity in the lateral line and embryotoxicity of zebrafish during embryonic development. Our results for TUNEL staining and DASPEI staining showed significantly increased apoptosis of hair cells as nicotine concentration increased. In the current study, the exposure of nicotine during zebrafish development seemed to increase the risk of embryotoxicity, caused signifi- 
cantly higher rates of abnormal morphology. However, hatching and mortality rates were higher in nicotine exposure groups than control group, but there was no significant dose-dependent trend. Nicotine is known to paralyze developing zebrafish when exposures occur during embryogenesis, and this leads to unhatching (paralyzed) or abnormal morphology (partially paralyzed) [25]. Our results partially correspond with previous studies that reported an increase in teratogenicity in a concentrationdependent manner following nicotine treatment $[3,8]$. Since inwell mortalities often cause increased toxicity to adjacent embryos, so this may have affected the increasing mortality as the nicotine concentration increase. However, there is a significant dose-dependent trend according to the nicotine concentration suggesting the teratogenic effect of nicotine.

Although heart rates were significantly lower in nicotine-exposed groups than the control group in our study, heart size was not different among groups and heart malformation was not observed in any of the groups. Our results for cardiac toxicity correspond with the findings of previous studies, which showed no heart defects in isolated nicotine exposure [9]. In contrast, when exposed to cigarettes, severe heart malformation including pericardial edema, reduced heart function was found in zebrafish $[8,9]$. Many chemicals in tobacco, including unknown and known ingredients such as polycyclic aromatic hydrocarbons are considered to cause cardiac toxicity in zebrafish [9]. Significant decrease in the heart rate of nicotine exposed zebrafish may have influenced on the development of the hair cells.

Nicotine is known to induce intracellular ROS generation in several kinds of cell lines and also in zebrafish [26,27]. Also in lateral line neuromasts of zebrafish, nicotine application provoked intracellular ROS generation which was demonstrated by our experiment. This ROS generation may have resulted in apoptosis of hair cells in neuromasts, which can be a possible mechanism of ototoxicity by nicotine.

Relatively few studies have investigated whether nicotine affects hearing in humans. In human population-based studies, current smoking [11], passive smoking [28] or past smoking [29] were all reported to be associated with hearing impairment after controlling for potential confounders. In addition, Durante et al. [15] reported that maternal tobacco exposure induced a significant decrease in transient evoked otoacoustic emissions amplitudes, suggesting an impact on outer hair cells. In a study of adolescents, maternal prenatal smoking exposure [30] or childhood nicotine exposure [14] were associated with higher puretone thresholds or abnormal transient evoked otoacoustic emissions amplitudes, respectively. Furthermore, few animal studies on this topic have been reported. In a study using rats, neonatal nicotine exposure reduced gap-induced prepulse inhibition suggesting damage of the normal development of auditory temporal processing by causing changes in cholinergic systems [16]. In the study of Abdel-Hafez et al. [18], nicotine caused structural damage of sensory hair cells, chiefly at the basal cochlear turn and outer hair cells, in adult guinea pigs. Especially, changes in stereocilia such as becoming bent and/or disorganized with a loss of their tip and side links were reported. Our SEM findings, which showed damage of kinocilia and the stereocilia bundles, correspond with those of Abdel-Hafez et al. [18]. Such damage of the hair cells and their stereocilia would result in clinical hearing loss, as recorded in humans [2,11].

Although several types of nAChRs have been described in zebrafish, the metabolism and half-life of nicotine in zebrafish are unknown [22]. We performed a literature review and pilot study to investigate the appropriate nicotine concentrations for the main research, and based on these findings used 5, 10, 20, 40 $\mu \mathrm{M}$ nicotine in the current study $[3,5,22]$. The mortality rate at $72 \mathrm{hpf}$ in the $40 \mu \mathrm{M}$ group was $9 \%$, compared with $19 \%$ for the $20 \mu \mathrm{M}$ group. However, when observed at $120 \mathrm{hfp}$, almost all of the fish in the $40 \mu \mathrm{M}$ nicotine group were dead (>90\% vs. $50 \%$ in $20 \mu \mathrm{M}$ group) and further statistical analysis for the $40 \mu \mathrm{M}$ group was not possible. This may be due to the high teratogenicity rate ( $44 \%$ for $40 \mu \mathrm{M}$ nicotine at $72 \mathrm{hpf}$ ) but was very low survival when compared with the study of Parker and Connaughton [3], which showed a survival rate of approximately $40 \%$ in the $40 \mu \mathrm{M}$ group at 5 days post-fertilization. Although the mortality rate at specific times should be compared between groups, surviving fish with abnormal morphology can also die at a later time therefore continuous monitoring is also very important.

We evaluated the hair cells in neuromasts at $120 \mathrm{hpf}$ after 120 hours of nicotine exposure. According to our previous unreported experiment in normal zebrafish, neuromasts at $72 \mathrm{hpf}$ (average of 6.8 hair cells per neuromast) and $96 \mathrm{hpf}$ (average of 8.8 hair cells per neuromast) are not fully developed and show fewer hair cells. At about 120 hpf, hair cell numbers reach normal range (average of 12.5 hair cells per neuromast) of hair cells in each neuromasts.

In conclusion, according to our knowledge, our findings are the first study to investigate nicotine-induced hair cell toxicity in the neuromasts of zebrafish. The current results suggest that nicotine induces dose-dependent mortality, teratogenicity, and hair cell toxicity through the induction of apoptosis and damage of stereocilia and kinocilia.

\section{CONFLICT OF INTEREST}

No potential conflict of interest relevant to this article was reported.

\section{ACKNOWLEDGMENTS}

This research was supported by a Korea University Grant (Seoul, Korea) and Soo ENT Clinic (Siheung, Korea). 


\section{REFERENCES}

1. Gray NJ. Nicotine yesterday, today, and tomorrow: a global review. Nicotine Tob Res. 2014 Feb;16(2):128-36.

2. Abbott LC, Winzer-Serhan UH. Smoking during pregnancy: lessons learned from epidemiological studies and experimental studies using animal models. Crit RevToxicol. 2012 Apr;42(4):279-303.

3. Parker B, Connaughton VP. Effects of nicotine on growth and development in larval zebrafish. Zebrafish. 2007 Spring;4(1):59-68.

4. Yoo MH, Rah YC, Choi J, Park S, Park HC, Oh KH, et al. Embryotoxicity and hair cell toxicity of silver nanoparticles in zebrafish embryos. Int J Pediatr Otorhinolaryngol. 2016 Apr;83:168-74.

5. Klee EW, Ebbert JO, Schneider H, Hurt RD, Ekker SC. Zebrafish for the study of the biological effects of nicotine. Nicotine Tob Res. 2011 May;13(5):301-12.

6. Zhao S, Cui WY, Cao J, Luo C, Fan L, Li MD. Impact of maternal nicotine exposure on expression of myelin-related genes in zebrafish larvae. Zebrafish. 2014 Feb;11(1):10-6.

7. Lantz-McPeak S, Guo X, Cuevas E, Dumas M, Newport GD, Ali SF, et al. Developmental toxicity assay using high content screening of zebrafish embryos. J ApplToxicol. 2015 Mar;35(3):261-72.

8. Ellis LD, Soo EC, Achenbach JC, Morash MG, Soanes KH. Use of the zebrafish larvae as a model to study cigarette smoke condensate toxicity. PLoS One. 2014 Dec;9(12):e115305.

9. Palpant NJ, Hofsteen P, Pabon L, Reinecke H, Murry CE. Cardiac development in zebrafish and human embryonic stem cells is inhibited by exposure to tobacco cigarettes and e-cigarettes. PLoS One. 2015 May;10(5):e0126259.

10. Welsh L, Tanguay RL, Svoboda KR. Uncoupling nicotine mediated motoneuron axonal pathfinding errors and muscle degeneration in zebrafish.Toxicol Appl Pharmacol. 2009 May;237(1):29-40.

11. Chang J, Ryou N, Jun HJ, Hwang SY, Song JJ, Chae SW. Effect of cigarette smoking and passive smoking on hearing impairment: data from a population-based study. PLoS One. 2016 Jan;11(1):e0146608.

12. Dawes P, Cruickshanks KJ, Moore DR, Edmondson-Jones M, McCormack A, Fortnum H, et al. Cigarette smoking, passive smoking, alcohol consumption, and hearing loss. J Assoc Res Otolaryngol. 2014 Aug;15(4):663-74.

13. Paschoal CP, Azevedo MF. Cigarette smoking as a risk factor for auditory problems. Braz J Otorhinolaryngol. 2009 Nov-Dec;75(6): 893-902.

14. Durante AS, Pucci B, Gudayol N, Massa B, Gameiro M, Lopes C.Tobacco smoke exposure during childhood: effect on cochlear physiology. Int J Environ Res Public Health. 2013 Oct;10(11):5257-65.

15. Durante AS, Ibidi SM, Lotufo JP, Carvallo RM. Maternal smoking during pregnancy: impact on otoacoustic emissions in neonates. Int J Pediatr Otorhinolaryngol. 2011 Sep;75(9):1093-8.

16. Sun W, Hansen A, Zhang L, Lu J, Stolzberg D, Kraus KS. Neonatal nicotine exposure impairs development of auditory temporal processing. Hear Res. 2008 Nov;245(1-2):58-64.

17. Talaat HS, Metwaly MA, Khafagy AH, Abdelraouf HR. Dose passive smoking induce sensorineural hearing loss in children? Int J Pediatr Otorhinolaryngol. 2014 Jan;78(1):46-9.

18. Abdel-Hafez AM, Elgayar SA, Husain OA, Thabet HS. Effect of nicotine on the structure of cochlea of guinea pigs. Anat Cell Biol. 2014 Sep;47(3):162-70.

19. Rah YC, Choi J, Yoo MH, Yum G, Park S, Oh KH, et al. Ecabet sodium alleviates neomycin-induced hair cell damage. Free Radic Biol Med. 2015 Dec;89:1176-83.

20. Chang J, Choi J, Rah YC, Yoo MH, Oh KH, Im GJ, et al. Sodium selenite acts as an otoprotectant against neomycin-induced hair cell damage in a zebrafish model. PLoS One. 2016 Mar;11(3):e0151557.

21. Kakehata S, Nakagawa T, Takasaka T, Akaike N. Cellular mechanism of acetylcholine-induced response in dissociated outer hair cells of guinea-pig cochlea. J Physiol. 1993 Apr;463:227-44.

22.Matta SG, Balfour DJ, Benowitz NL, Boyd RT, Buccafusco JJ, Caggiula AR, et al. Guidelines on nicotine dose selection for in vivo research. Psychopharmacology (Berl). 2007 Feb;190(3):269-319.

23. Ackerman KM, Nakkula R, Zirger JM, Beattie CE, Boyd RT. Cloning and spatiotemporal expression of zebrafish neuronal nicotinic acetylcholine receptor alpha 6 and alpha 4 subunit RNAs. Dev Dyn. 2009 Apr;238(4):980-92.

24.Thomas LT, Welsh L, Galvez F, Svoboda KR.Acute nicotine exposure and modulation of a spinal motor circuit in embryonic zebrafish. Toxicol Appl Pharmacol. 2009Aug;239(1):1-12.

25. Svoboda KR, Vijayaraghavan S, Tanguay RL. Nicotinic receptors mediate changes in spinal motoneuron development and axonal pathfinding in embryonic zebrafish exposed to nicotine. J Neurosci. 2002 Dec;22(24):10731-41.

26. Rao P, Ande A, Sinha N, Kumar A, Kumar S. Effects of cigarette smoke condensate on oxidative stress, apoptotic cell death, and HIV replication in human monocytic cells. PLoS One. 2016 May;11(5): e0155791.

27. Nathiga Nambi KS, Abdul Majeed S, Taju G, Sivasubbu S, Sarath Babu V, Sahul Hameed AS. Effects of nicotine on zebrafish: a comparative response between a newly established gill cell line and whole gills. Comp Biochem Physiol C Toxicol Pharmacol. 2017 May;195:68-77.

28. Fabry DA, Davila EP, Arheart KL, Serdar B, Dietz NA, Bandiera FC, et al. Secondhand smoke exposure and the risk of hearing loss. Tob Control. 2011 Jan;20(1):82-5.

29. Mustafa MW.Transient evoked otoacoustic emissions and vestibular evoked myogenic potentials in cigarette and water pipe smokers. Eur Arch Otorhinolaryngol. 2014 Oct;271(10):2669-73.

30. Weitzman M, Govil N, Liu YH, Lalwani AK. Maternal prenatal smoking and hearing loss among adolescents. JAMA Otolaryngol Head Neck Surg. 2013 Jul;139(7):669-77. 